\title{
Estimación de la Edad Médico-Legal Usando dos Métodos para la Medición de la Translucidez Dentinaria Radicular: Análisis Comparativo
}

\author{
Legal Medical Age Estimation Using Two Methods Measuring \\ Root Dentin Translucency: Comparative Analysis
}

Patricio Carrasco Tapia*; Joaquín González Srur*; Claudia Brizuela Cordero* \& Carolina Inostroza Silva*

\begin{abstract}
CARRASCO, T. P.; GONZÁLEZ, S. J.; BRIZUELA, C. C. \& INOSTROZA, S. C. Estimación de la edad médico-legal usando dos métodos para la medición de la translucidez dentinaria radicular: Análisis comparativo. Int. J. Morphol., 32(3):956-961, 2014.

RESUMEN: En una investigación forense existen diversas incógnitas médico legales y criminalísticas por definir: especie, sexo, edad, identidad, causa y data de muerte de la víctima o victimario. Fenómenos biológicos han colaborado en estimar la edad médico legal de un individuo. Investigadores han propuesto diferentes métodos para esta estimación. El fenómeno de la translucidez dentinaria radicular se asocia con el aumento de la edad del individuo y ha demostrado ser de gran utilidad en esta estimación. El objetivo fue comparar el acierto en la estimación de la edad médico legal en tres rangos etarios predeterminados, mediante la medición de la translucidez dentinaria radicular, aplicando dos metodologías. Se realizaron mediciones de la longitud de la translucidez de piezas dentarias de una población chilena $(\mathrm{n}=94)$, mediante un método visual directo convencional y un método digital indirecto basado en una captura computarizada de la imagen. Se estimó la edad aplicando una tabla propuesta. Se preestablecieron tres rangos de edad: 1er rango (20-39 años), 2do rango (40-59 años) y 3er rango (60-79 años). El método digital muestra un acierto en la estimación de la edad en el 1er rango etario (93\%), en comparación al método visual que muestra un acierto 2do y 3er rango (86\% y 65\%). Existen diferencias significativas $\mathrm{p}=0,001$ entre ambos métodos para cada rango. La diferencia no es estadísticamente significativa en los porcentajes totales de aciertos en la estimación de la edad con ambos métodos. Enfrentados a la estimación de la edad en piezas dentarias con poca TDR se recomienda el método digital, considerando que tiende a una subestimación. En casos de mayor TDR recomendamos el método visual, que tiende a sobreestimar. Este trabajo demuestra que ambos métodos son igualmente efectivos.
\end{abstract}

PALABRAS CLAVE: Estimación de edad; Translucidez dentinaria radicular; Identificación médico legal; Odontología forense.

\section{INTRODUCCIÓN}

La identificación médico legal se define como el procedimiento científico forense realizado en personas vivas o fallecidas (restos parciales o totales cadavéricos), involucrados en un proceso judicial, con el objeto de reconocer la identidad. De esta manera se colabora con el juez en la aplicación de justicia (Carrasco et al. 1993).

Cuando en un proceso judicial es necesario determinar la identidad de un individuo, la estimación de la edad es fundamental. La determinación de la edad en cuerpos o restos cadavéricos desconocidos es un problema frecuente en Medicina Legal. De importancia no solo por los alcances judiciales que esto conlleva sino también porque es un aspecto básico en el proceso de la identificación médico legal.
La determinación de la edad Médico Legal se transforma en un verdadero desafío cuando los restos humano han estado sujetos a procesos de putrefacción, combustión y otras fuerzas externas. En muchos de estos casos, lo único que se preservaron las estructuras duras: hueso y dientes, y por ello, estos últimas podrían ser el único recurso para determinar la edad médico legal es decir, la edad que tenía al momento de fallecer (Carrasco et al.).

Diversos métodos se han propuesto para calcular la edad utilizando los dientes, desde la correlación con procesos de crecimiento y desarrollo (bajo 20 años de edad) hasta fenómenos de tipo regresivos de las estructuras del diente (sobre 20 años de edad), tales como atrición de esmalte, dentículos pulpares, apósitos de cemento, erupción conti 
nua, periodontopatías, translucidez dentinaria radicular e incluso señales bioquímicas (Gustafson, 1950; Nalbandian et al., 1960; Bang \& Ramm, 1970; Lamendin et al., 1992).

En la literatura se ha descrito la relación entre la translucidez dentinaria radicular y el aumento de edad en individuos sobre 20 años de edad, pero, aun así no se ha logrado consenso en establecer un método único, confiable y preciso que entregue un resultado satisfactorio, ya sea por la existencia de factores subjetivos, falta de personal especializado, infraestructura inadecuada, tecnológica utilizada no validada, entre otros (Kinney et al., 2003).

Carrasco et al. (1992) crearon una tabla de determinación de edad a través de la medición de la translucidez dentinaria radicular (TDR) mediante un método visual convencional sobre una muestra de 57 dientes de población chilena, a partir del cálculo de regresión lineal entre TDR y edad real, logrando una estimaciones de edad con un $70,8 \%$ de certeza con un \pm 6 años. Los datos provenían del cálculo de regresión lineal de la muestra estudiada basado en la metodología descrita por Bang et al. 1970. Se concluyó que el método propuesto por Bang et al. 1970, es útil para determinar la edad médico legal en individuos mayores a 20 años, cuantificando la translucidez dentinaria radicular; a través del uso de tablas para dientes indemnes y ecuaciones de regresión de 1er y 2 do grado. Según los resultados, de la cuantificación de las diferencias entre edad real y edad estimada, según Bang et al., a los \pm 6 años se logró un 64,56\% de acierto en la edad correcta, mientras que con el método chileno de regresión lineal, a los \pm 6 años, se logró una certeza del 70,83\%.

En base a los buenos resultados obtenidos a partir del cálculo de regresión lineal entre TDR y edad real, se determinó que la tabla chilena para la determinación de la edad médico legal era muy confiable (Carrasco et al. 1992). En dicha tabla se estableció que cada 0,5 de $\mathrm{mm}$ de translucidez radicular se estimaban tres años de edad, determinándose como límite inferior de longitud de translucidez radicular valores menores a 0,5 mm para los 20 años de edad. Este cálculo se realizó en base a la fórmula: $Y=-3,425+0,176 \mathrm{x}$ " $\mathrm{X}$ ", con un $\mathrm{r}=0,904$. La tabla descrita en ese trabajo sirvió de instrumento de estimación de edad en este estudio. Ramsthaler et al. (2014), utilizó una metodología digital para la medición de la TDR en dientes seccionados, metodología que posee la ventaja de ser operador independiente.

Considerando la posibilidad de que la medición visual directa se ve afectada por variables operador dependiente, propusimos la aplicación de un método de medición digital para mejorar la reproducibilidad, disminuir la variabilidad entre operadores distintos y determinar la edad médico legal con mayor precisión. Enfrentados a la problemática de resolver identificaciones de un gran número de restos humanos fragmentados en un desastre masivo, consideramos relevante en una primera aproximación agrupar los restos de acuerdo al siguiente criterio: jóvenes, adultos o adultos mayores para facilitar el proceso identificatorio de un gran número de víctimas. Utilizando este criterio hemos preestablecido tres rangos etarios.

\section{MATERIAL Y MÉTODO}

Se recolectaron las muestras a utilizar, desde un banco forense de dientes, formado desde al año 2011 en el Laboratorio de Investigaciones Odontológicas (CIBRO) de la Universidad de los Andes, que cumple con los requisitos exigidos en el artículo 2 de la ley sobre Investigación Científica en el Ser Humano (Ley N 20.120) y los artículos 21 y 22 de la Ley de Deberes y Derechos del Paciente (Ley N 20.5849).

El total de dientes seleccionados $(n=94)$ de ambos sexos, correspondía a dientes uni o multiradiculares con indicación de exodoncia, ápice radicular indemne de donantes de más de 20 años de edad; con consentimiento informado firmado. La obtención y la manipulación de las muestras humanas se realizaron bajo las consideraciones exigidas por el Comité de Bioética de la Universidad de los Andes.

En este estudio, de acuerdo a los objetivos planteados se decidió pre establecer los siguientes rangos etarios: Rango 1: 20-39 años; Rango 2: 40-59 años y Rango 3: 60 años o mayor. Estos rangos nos permiten una vez estimada la edad agrupar las piezas dentarias de sujetos jóvenes, adultos y adulto mayor.

Se utilizaron dos métodos para la observación y medición de la longitud de la TDR. El método digital indirecto, consistió en instalar el diente en un sistema de foto documentación de geles de agarosa (Enduro GDS, Labnet International Inc.), cuya característica fundamental es que permite la instalación de un negatoscopio de luz led como fuente de luz constante, fría y uniforme, además de incluir una cámara científica de resolución 5MP para la adquisición de imágenes que posteriormente son almacenadas en un software (Enduro GDS, Aplegen). La imagen tomada en el sistema de foto documentación se realizó con un Zoom X5, luz de $1 \mathrm{msec}$ y contraste definido (Black: 0, White 65535, Gamma 2,20).

El método convencional directo, consiste en una medición visual directa de la TDR de dientes mediante la colocación de ella en una fuente de luz constante, fría y uniforme (negatoscopio, especialmente diseñado). 
Ambas metodologías se realizaron en un cuarto oscuro para evitar que el exceso de luz ambiental interfiriera de algún modo en las mediciones. Los resultados de la longitud de la TDR se realizaron utilizando una misma regla forense blanco-negro 105 mm x 105 mm (PPS800 Sirchie Finger Print Laboratoriestm). Esta regla PPS800 está diseñado principalmente para profesionales forenses que están involucrados con el estudio de la anatomía, el crecimiento y las patologías dentales. La escala se imprimió en vinilo rígido, plástico mate y cuenta con excelente definición de la división.

La regla se colocó adyacente al diente previamente fijado con cera en su porción coronal. En ambos métodos se midió en centímetros la longitud de la translucidez radicular observada, es decir, la distancia comprometida entre el ápice indemne de la pieza dentaria hasta la zona más coronal de la translucidez observada.

Análisis estadístico. Obtenida la medición de la TDR en todos los dientes seleccionados con ambos métodos, se procedió a estimar la edad del individuo, aplicando la Tabla I para la estimación de edad confeccionada por Carrasco et al. (1992). Se realizó el análisis estadístico de los datos obtenidos mediante el software estadístico "STATA" versión 11.2 para Windows (Stata Corp LP, Texas, USA). Se determinó la proporción de concordancias (índice de Cohen, Kappa) entre las edades estimadas mediante ambos métodos y el tipo de pieza dentaria utilizada. Además, se obtuvo el grado de concordancia según el valor del coeficiente de Lin entre la medición de la edad obtenida mediante el método digital y el método visual. Se determinó la distribución porcentual del acierto en la determinación de la edad en la muestra según ambos métodos.

\section{RESULTADOS}

El método digital mostró mejores resultados en la estimación de edad en el 1er rango etario (93\%), en comparación al método visual que fue más asertivo en el 2do y 3er rango $(86 \%$ y $65 \%)$. Existen diferencias significativas $\mathrm{p}=0,001$ entre ambos métodos para cada rango etario (Fig. 1).

Debido a que en la muestra tenemos 4 tipos de dientes: incisivos, caninos, premolares y molares; determinamos la proporción de concordancias de las edades estimadas (índice de Kappa) obtenidas mediante los dos métodos en relación con el tipo de diente. En la Figura 2 se muestra que la concordancia según el método visual es de tipo "moderada" (41\%-60\%) en piezas incisivos y caninos. Sin embargo, para este mismo método existe una concordancia de tipo "sustancial" (61\%-80\%) en dientes premolares y molares. El método digital presenta una concordancia mediante el índice de Kappa de tipo "sustancial" en todos los tipos de dientes.

\begin{tabular}{|l|l|l|l|l|l|l|l|l|l|l|l|l|l|l|l|l|l|l|l|l|l|l|l|l|l|l|l|}
\hline & \multicolumn{2}{|c|}{ Edad en años } \\
\hline
\end{tabular}

Tabla I. Determinación de la edad médico legal a través de la medición de la longitud de la translucidez dentinaria (regresión lineal) de una población chilena. Tomada de Carrasco et al. (1992). 


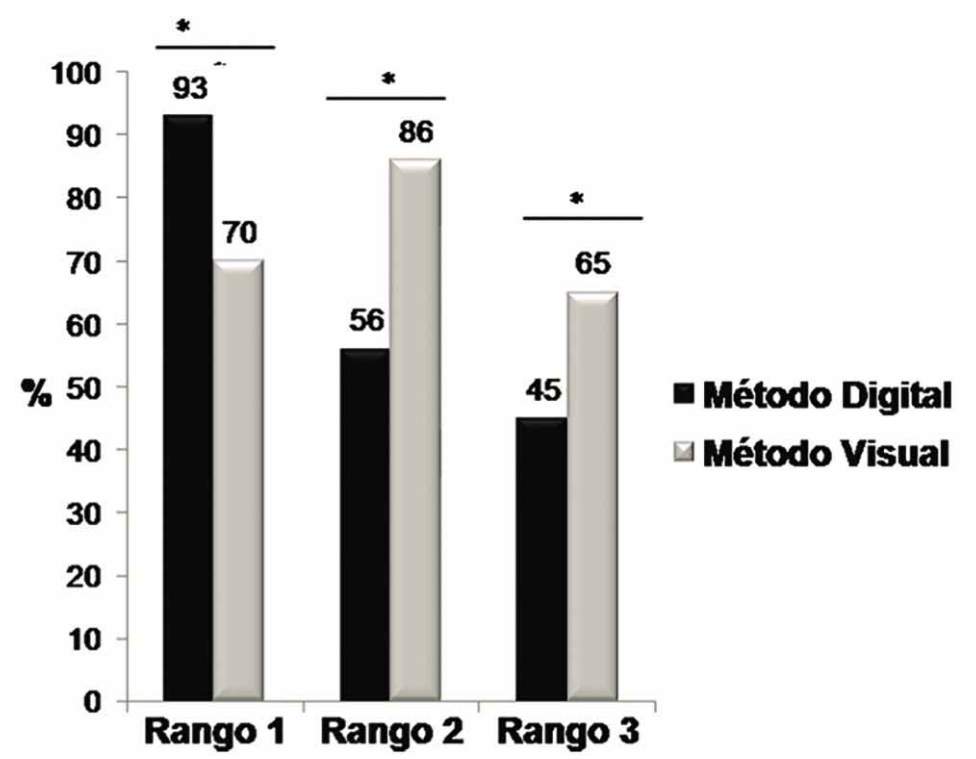

Fig. 1. Distribución porcentual del acierto en la determinación de la edad según los rangos etarios predeterminados mediante el uso de ambos métodos. Se determinó el acierto en ambos métodos (digital indirecto y visual directo) en 3 rangos: rango 1 (20-39 años), rango 2 (40-59 años) y rango 3 (60 años o más). Obtenemos una diferencia significativa entre ambos métodos para cada rango etario $(* \mathrm{p}=0,001)$.

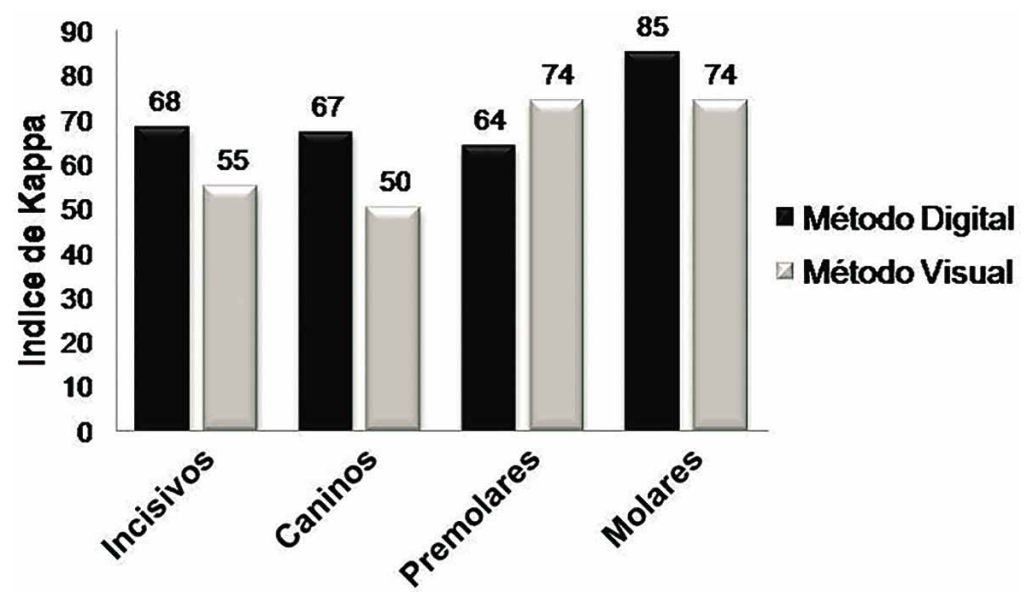

Fig. 2. Proporción de concordancias de las edades estimadas (índice de Kappa) obtenidas mediante los métodos utilizados (visual y digital) en relación con el tipo de diente. Se muestra la concordancia mediante el índice de Kappa obteniéndose en el método visual una concordancia de tipo "moderada" (41-60\%) en piezas incisivos y caninos, y una concordancia de tipo "sustancial" en dientes premolares y molares. El método digital presenta una concordancia mediante el índice de Kappa de tipo "sustancial" (61-80\%) en todos los tipos de dientes.

La distribución de las edades estimadas según ambos métodos en relación a la edad real muestra que al utilizar el método digital la edad obtenida tiende a subestimarse (Fig. 3A) y al utilizar el método visual la edad obtenida tiende a sobreestimarse (Fig. 3B). La diferencia entre los porcentajes totales de aciertos en la estimación de la edad obtenidos con ambos métodos, no es estadísticamente significativo (Fig. 4).

\section{DISCUSIÓN}

En el proceso de identificación médico legal de individuos adultos, mediante el uso de dientes, una de las incógnitas que es necesario estimar y que colabora de manera considerable al reconocimiento de la identidad, es la edad, es decir si los restos humanos en estudio corresponden a un joven, adulto o adulto mayor.

Distintas investigadores han estimado la edad mediante el uso del método visual directo de la translucidez dentinaria radicular (Bang \& Ramm, 1970; Drusini et al., 1991a, 1991b; De Jonge, 1950; de las Heras et al., 2005). Aunque el fenómeno de la TDR es conocido, aun no existe un consenso para la definición de una metodología única.

Recientemente Ramsthaler et al., realizaron un análisis comparativo de dos métodos para medir la TDR, visual directo convencional y digital computarizado indirecto. La muestra analizada correspondía a secciones longitudinales de dientes. En este estudio se sugiere utilizar la metodología digital por ser una técnica normalizada (luz uniforme, técnica digital de medición, baja dependencia de un operador experimentado).

En nuestro trabajo se predeterminaron 3 rangos de edad, un primer rango que comprende desde los 20 a los 39 años, un segundo rango desde los 40 a los 59 años y un tercer rango que comprende individuos de 60 años o más. La razón de esta clasificación está dada por el interés en conocer cuál de los dos métodos se desenvuelve de mejor forma enfrentado a la identificación de un gran número de individuos comprometidos en un desastre masivo, en el cual es necesario en una primera etapa clasificar los restos humanos en: jóvenes, adultos y adultos mayores.

$\mathrm{Al}$ analizar la distribución porcentual del acierto en la determinación de la edad en los rangos predeterminados, se observó que existe una diferencia significativa entre ambos métodos en todos los rangos etarios $(\mathrm{P}=0,001)$. 

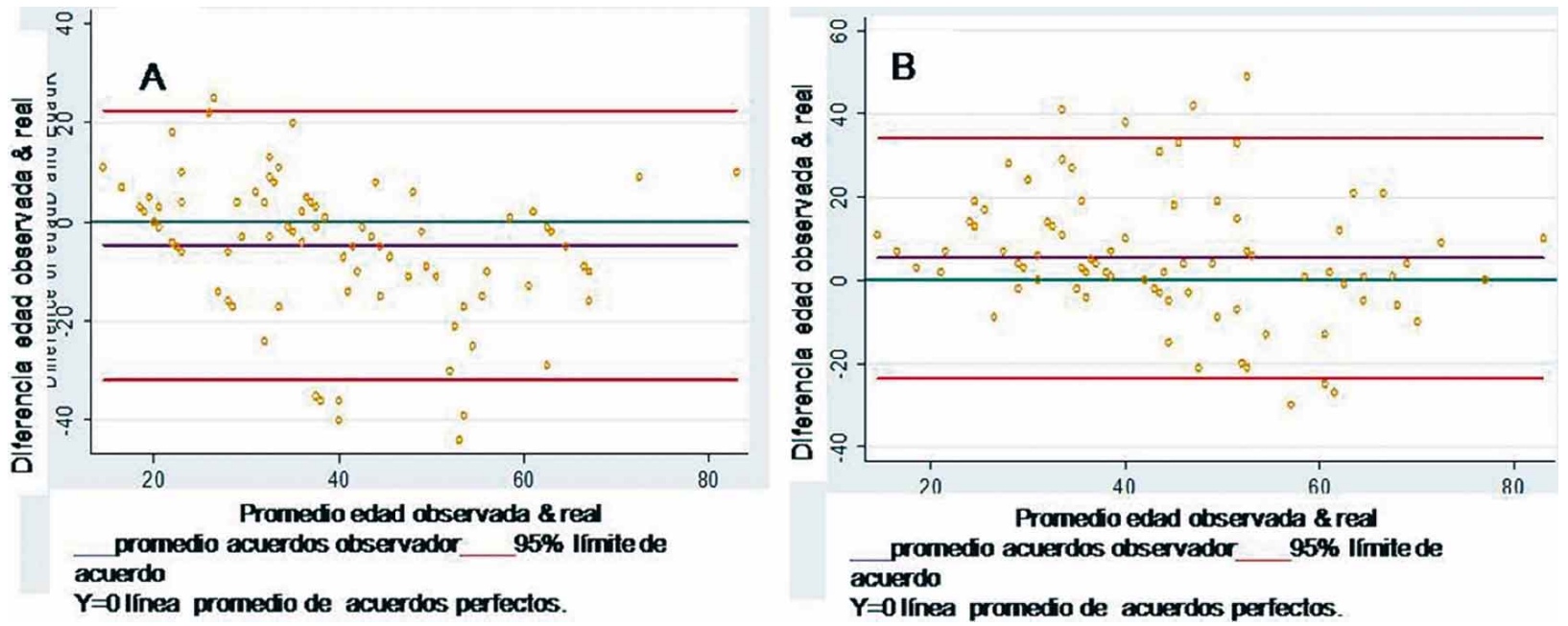

Fig. 3. Distribución de las edades estimadas según ambos métodos en relación a la edad real. En los gráficos A y B se observa la distribución de las edades estimadas mediante el método digital (A) y método visual (B) en relación a la edad real. Los puntos representan todos los casos, aquellos que se encuentran próximos a las líneas centrales corresponden a edades más certeras, mientras que aquellos casos que se escapan de la línea periférica inferior corresponden a aquellos casos en la cual la edad estaría subestimada (A) aquellos casos que se escapan de la línea periférica superior corresponden a aquellos casos en la cual la edad estaría sobreestimada (B).

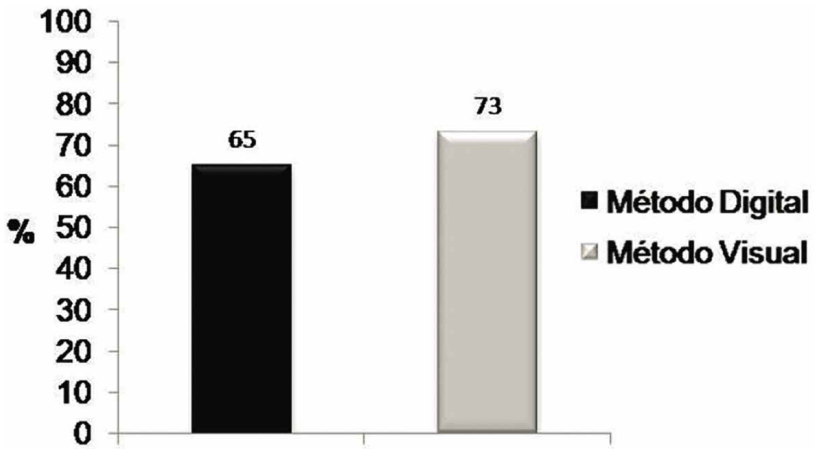

Fig. 4. Distribución porcentual del acierto en la determinación de la edad en la totalidad de la muestra según ambos métodos. En este gráfico se muestra un porcentaje de $65 \%$ en la estimación de la edad obtenida con el método digital indirecto. El porcentaje de acierto fue de un $73 \%$ para la estimación de la edad obtenida con el método visual directo. Los resultados se expresan como porcentajes en valores absolutos y no existen estadísticamente diferencias significativas entre ambos métodos.

En el rango 1 existe diferencia significativa a favor del método digital, mientras que para el rango 2 y 3 existe una diferencia significativa a favor del método visual (Fig. 1).

En relación a la distribución porcentual del acierto en la estimación de la edad según el tipo de diente, el método visual directo presenta mejores resultados en dientes anteriores (incisivos y caninos). Este resultado puede deberse a la menor incertidumbre que se genera en el operador al analizar un diente unirradicular, comparado con las dificultades que puede darse en un diente multirradicular. La esti- mación de la edad con ambos métodos según el tipo de diente, no presentó diferencias significativas en el acierto (Fig. 2). Cabe destacar que el resultado obtenido con el uso del método visual directo en esta investigación (acierto 73\%) presenta una concordancia con los resultados obtenidos por Carrasco et al. (1992) (acierto 70,8\%).

Si bien se observó una concordancia y acierto en la estimación de la edad entre ambos métodos por separado. Sin embargo, se logró observar que de acuerdo a los índices de concordancia, el método digital indirecto tiende a subestimar la edad principalmente en el segundo rango etario, es decir tiende a estimar edades menores a la edad real. El método visual directo por el contrario, tiende a sobrestimar la edad y también lo hace principalmente en el segundo rango etario, es decir estima edades mayores a la reales (Fig. 3).

$\mathrm{Al}$ realizar un análisis comparativo entre los métodos utilizados con respecto a la estimación de la edad, no se encontró una diferencia significativa que afirme la eficacia de un método por sobre el otro. La diferencia entre los porcentajes totales de aciertos en la estimación de la edad obtenidos con ambos métodos, no es estadísticamente significativo (Fig. 4).

Nuestro estudio coincide con los resultados obtenidos por Singh et al. (2013), quien sugiere aumentar el tamaño de la muestra para determinar cuál de los dos métodos es más confiable y certero. Cabe destacar que esta investigación referida a TDR y su aplicación en la estimación de la edad médico legal utilizó la Tabla I generada con muestras dentarias de población chilena. 
La relevancia de esta investigación radica en que una vez estimada la edad y por ende el rango etario, se podrían agrupar los restos humanos involucrados en un desastre masivo, en cuerpos de individuos: jóvenes, adultos y adultos mayores, para posteriormente continuar con el proceso de identificación forense.
AGRADECIMIENTOS. Agradecemos a la Universidad de los Andes por el apoyo y financiamiento otorgado: Proyecto FIC ODO 2013, Facultad de Odontología. Proyecto FAI ODO 2012, Dirección de Investigación.

CARRASCO, T. P.; GONZÁlez, S. J.; BRIZUElA, C. C. \& INOSTROZA, S. C. Legal medical age estimation using two methods measuring root dentin translucency: Comparative analysis. Int. J. Morphol., 32(3):956-961, 2014.

SUMMARY: In a forensic investigation there are several forensic and criminology unknowns to be elucidated, such as, species, sex, age, identity, cause and date of death of the victim or perpetrator. Some biological phenomena have been used to collaborate in determining the forensic age of an individual. Many authors and researchers have proposed different ways to perform this estimate. The phenomenon of root dentine translucency (RDT) is associated with the increase of age of the individual and has proven to be useful in the estimation of this unknown. The objective of this study was to compare the accuracy in estimating forensic age in three age ranges, by measurement of RDT, using two different methodologies. The protocol consists of taking measurements of the length translucency of dental pieces in a Chilean population $(n=94)$, by direct visual method conventionally used and an indirect digital method based on the capture of a computerized image. The age was estimated applying a table: Three age ranges are preset: 1st range (20-39 ages), 2nd range (40-59 ages) and 3rd range (60-79 ages). The digital method gave better results in the estimation of age in the 1st age range (93\%), compared to the visual method that was more assertive in the 2 nd and 3rd range ( $86 \%$ and $65 \%$ ). There are significant differences $\mathrm{p}=0.001$ between the methods for each age range. The difference between the total percentages of successes in the age estimate obtained with both methods are not statistically significant. In conclusion, for age determination in teeth with very little translucency the digital method is recommended, although we must consider that this method tends to underestimate age. In cases of higher RDT we recommend using the visual method; however this method tends to overestimate age. Regardless of the above conclusion, this work shows that both methods are equally effective.

KEY WORDS: Age estimation; Root dentine translucency; Forensic identification; Forensic dentistry.

\section{REFERENCIAS BIBLIOGRÁFICAS}

Bang, G. \& Ramm, E. Determination of age in humans from root dentin transparency. Acta Odontol. Scand., 28(1):3-35, 1970.

Carrasco, P.; Hernández, P. \& Mery, A. J. Determinación de la edad médico legal en niños entre 5 y 13 años. Proposición de una tabla. Odontol. Chil., 41(1):23-31, 1993.

Carrasco, P.; Muñoz, L. \& Mery, J. Determinación de la edad médico legal a través del estudio de la transparencia dentinaria radicular en piezas dentarias indemnes. Comprobación de un método. Trabajo de investigación para optar por el título de cirujano dentista. Facultad de Odontología, Universidad de Chile, 1992.

Drusini, A. G. Age-related changes in root transparency of teeth in males and females. Am. J. Hum. Biol., 3(6):626-37, 1991.

Drusini, A.; Calliari, I. \& Volpe, A. Root dentine transparency: age determination of human teeth using computerized densitometric analysis. Am. J. Phys. Anthropol., 85(1):25-30, 1991 b.

De Jonge, T. E. Das Altern des Gebisses. Parodontologie, 4:83-98, 1950.

de las Heras, S. M. Estimación de la edad a través del estudio dentario. Cienc. Forense: Rev. Aragon. Med. Leg., 7:69-90, 2005.

Gustafson, G. Age determination on teeth. J. Am. Dent. Assoc., 41(1):45$54,1950$.

Kinney, J. H.; Marshall, S. J. \& Marshall, G. W. The mechanical properties of human dentin: a critical review and re-evaluation of the dental literature. Crit. Rev. Oral Biol. Med., 14(1):13-29, 2003.

Lamendin, H.; Baccino, E.; Humbert, J. F.; Tavernier, J. C.; Nossintchouk, R. M. \& Zerilli, A. A simple technique for age estimation in adult corpses: the two criteria dental method. $J$. Forensic Sci., 37(5):1373-9, 1992.

Nalbandian, J.; Gonzales, F. \& Sognnaes, R. F. Sclerotic age changes in root dentin of human teeth as observed by optical, electron, and x-ray microscopy. J. Dent. Res., 39:598-607, 1960.

Ramsthaler, F.; Kettner, M. \& Verhoff, M. A. Validity and reliability of dental age estimation of teeth root translucency based on digital luminance determination. Int. J. Legal Med., 128(1):171-6, 2014.

Singh, S.; Venkatapathy, R.; Balamurali, P.; Charles, N. \& Suganya, R. Digital approach for measuring dentin translucency in forensic age estimation. J. Forensic Dent. Sci., 5(1):47-51, 2013.

Dirección para Correspondencia:

Prof. Dr. Patricio Carrasco Tapia

Universidad de los Andes

Odontología Legal

Mons. Álvaro del Portillo 12.455

Las Condes, Santiago

CHILE

Email: pcarrasco@miuandes.cl

Recibido : 23-12-2013 Aceptado: 02-05-2014 